\title{
HYBRID REDUCED ORDER MODELING FOR ASSEMBLY CALCULATIONS
}

\author{
Youngsuk Bang \\ FNC Technology, Co. Ltd., \\ South Korea \\ ysbang00@fnctech.com, \\ Hany S. Abdel-Khalik \\ Purdue University \\ West Lafayette, Indiana, USA \\ abdelkhalik@purdue.edu, \\ Matthew A. Jessee and Ugur Mertyurek \\ Oak Ridge National Laboratory \\ Oak Ridge, Tennessee, USA \\ jesseema@ornl.gov, mertyurek@ornl.gov
}

\begin{abstract}
While the accuracy of assembly calculations has considerably improved due to the increase in computer power enabling more refined description of the phase space and use of more sophisticated numerical algorithms, the computational cost continues to increase which limits the full utilization of their effectiveness for routine engineering analysis. Reduced order modeling is a mathematical vehicle that scales down the dimensionality of large-scale numerical problems to enable their repeated executions on small computing environment, often available to end users. This is done by capturing the most dominant underlying relationships between the model's inputs and outputs. Previous works demonstrated the use of the reduced order modeling for a single physics code, such as a radiation transport calculation. This manuscript extends those works to coupled code systems as currently employed in assembly calculations. Numerical tests are conducted using realistic SCALE assembly models with resonance self-shielding, neutron transport, and nuclides transmutation/depletion models representing the components of the coupled code system.
\end{abstract}

Key Words: Reduced Order Modeling, Multi Physics, Reactor Physics

\section{INTRODUCTION}

To properly model the evolution of neutrons inside a reactor core, one must consider the different physics affecting neutron multiplication. Given the complexity of the associated physics models, an operator split approach has been adopted in reactor physics calculations. This means that only one physics is updated at a time, while keeping all other physics constant. For example, the neutron transport equation is used to calculate the neutron flux, assuming constant cross-sections and isotopic concentrations. Though this approach enables modeling each physical process in details under the prescribed conditions, it brings other issues in aspects of interactions and feedbacks between models. For example, as fission reactions occur, the isotopic concentrations 
are changed in different rates, which alters the medium temperature distribution. This changes reaction probabilities due to resonance self-shielding and Doppler effects and eventually feeds back to reaction rates.

In conventional reactor physics calculations, intricate coupling techniques employing detailed look-up tables have been developed over the years to account for these complex interactions. First, the assembly calculations are conducted for different operating conditions (e.g. enrichments, rod insertion history, temperatures or densities) and the calculated group constants (e.g. cross sections, assembly discontinuity factors, form factors) are tabulated. Then, the full core calculations are performed with group constants adjusted by interpolating those tabulated ones. Compared to other methods, e.g. tight integration, this approach requires least effort to incorporate multi-physics models; however, it may be tedious to prepare look-up tables, which may take several thousands of assembly calculations. Therefore, the reduction of the computational time would be beneficial in engineering applications, e.g. sensitivity analysis, uncertainty quantification or design optimization.

Reduced Order Model (ROM) identifies the dominant features to reduce the effective dimensionality of the model, thereby forming the more compact representation. These features can be expressed as linear combinations of the original model parameters, which is described mathematically by a basis of the influential subspace. The primary objective of ROM is to seek a minimal size of a basis that captures all essential features in order to meet a user-defined error tolerance for the reduced model. Several approaches have been proposed and used in areas of system and control theory, computational fluid dynamics, and electro-mechanical analysis [1]. In nuclear engineering, efficient basis construction algorithms based on randomized subspace methodologies have been proposed and their feasibility to reactor physics applications has been demonstrated. For example, the state-level reduction technique was incorporated into initial condition perturbation theory to reduce the number of adjoint mode runs [2]. The parameter-level reduction technique was applied to second order sensitivity analysis and uncertainty propagation [3]. It is important to note that the dominant linear/nonlinear behavior of a model could be preserved accurately within a given error tolerance but these developments were limited to single-physics problems.

This manuscript extends past developments to a multi-physics model in which models are serially coupled by one-way data transfer. By performing a second reduction at the interface between two physics models, using the concept of subspaces intersection, one can further reduce the effective dimensionality of the overall multi-physics model. In this manuscript, we focus on an assembly model as an example of a multi-physics model, wherein resonance calculations, radiation transport, and depletion calculations represent three coupled physics models. The essential basis for the resonance self-shielded macroscopic cross sections which are the main data transferred from resonance calculations to radiation transport calculations is extracted and its completeness for capturing overall model behaviors is examined.

\section{MATHEMATICAL DESCRIPTION}

The basic idea of reduced order modeling is to build a compact model preserving only essential features in model behaviors. Identifying those features can be mathematically described as basis 
construction. In this section, the previous reduced order modeling methods are reviewed which are for a single model. In addition, the efficient basis construction algorithm, i.e. a randomized range finder, is briefly explained. Then, a procedure for identifying the so-called intersection basis for serially coupled codes is proposed to achieve further reduction.

\subsection{Mathematical Model}

Suppose a single physics is described by:

$$
T(\vec{p}, \vec{\phi})=\zeta(\vec{p})
$$

where $\vec{p} \in \mathbb{R}^{n}$ is a vector of $n$ input parameters and $\vec{\phi} \in \mathbb{R}^{k}$ denotes a state vector. $T$ is a general nonlinear function depends on input parameters and state variables. $\zeta$ is a direct contribution of the parameters to the governing equations as well as forcing due to boundary conditions and source terms. The input parameters are often measured experimentally or generated using preprocessor models; they represent the independent variables for the model. The state variables is a solution of the model, i.e. Eq. (1). Once the state solution is obtained, the responses can be readily calculated as general functions of the state and the parameters.

\subsection{Reduced Order Modeling in Two Levels}

Reduced order modeling attempts to find an accurate approximation of model behaviors with fewer degrees of freedom. Different approaches can be considered depending on where the reduction is rendered: state-level and parameter-level.

The state-level reduction assumes that the variability of the state can be well approximated by a subspace spanned by a reduced basis. In this case, the computational cost to solve the reduced order model becomes dependent on the size of the reduced basis rather than the original state space. This idea has led to tremendous savings in computational overhead especially for complex models where a detailed description of the state must be calculated. Mathematically, this reduction can be described by re-writing Eq. (1) as follows:

$$
T\left(\vec{p}, \mathbf{Q}_{\phi} \mathbf{Q}_{\phi}^{T} \vec{\phi}\right)=\zeta(\vec{p})
$$

This representation implies that a subspace spanned by $\mathbf{Q}_{\phi} \in \mathbb{R}^{k \times d_{k}}$ captures all essential state variations. To render a reduced system of equations, projection methods employ a further projection of Eq. (2) onto a subspace of $\mathbf{Q}_{\phi}$ :

$$
\mathbf{Q}_{\phi}^{T} T\left(\vec{p}, \mathbf{Q}_{\phi} \tilde{\phi}\right)=\mathbf{Q}_{\phi}^{T} \zeta(\vec{p})
$$


where $\tilde{\phi}=\mathbf{Q}_{\phi}^{T} \vec{\phi} \in \mathbb{R}^{d_{k}}$ with $d_{k}<k$. Note that this type of reduction does not make any implicit assumptions about the degree of nonlinearity of the model. Although the transformation between the original variables and reduced variables is linear, the reduced order model equations would still preserve nonlinear behaviors but over a reduced number of dimensions.

If the reduction is exercised at the parameter-level, one needs to identify a subspace of parameters which results in variations in the state and the responses. In other words, all input parameter perturbations that are orthogonal to this subspace produce negligible variations in the state and the responses. To identify such subspace, one must have access to the first order derivatives of the responses with respect to the parameters, i.e. gradient information [3]. Mathematically, this reduction may be described by re-writing Eq. (1) as follows:

$$
T\left(\mathbf{Q}_{p} \mathbf{Q}_{p}^{T} \vec{p}, \vec{\phi}\right)=\zeta\left(\mathbf{Q}_{p} \mathbf{Q}_{p}^{T} \vec{p}\right)
$$

In this representation, a subspace of $\mathbf{Q}_{p}$ inside the parameter space is assumed to represent all parameter perturbations. The reduced input parameters can be defined as:

$$
\tilde{p}=\mathbf{Q}_{p}^{T} \vec{p}
$$

where $\tilde{p} \in \mathbb{R}^{d_{n}}$. Then, Eq. (4) can be re-written as:

$$
T\left(\mathbf{Q}_{p} \tilde{p}, \vec{\phi}\right)=\zeta\left(\mathbf{Q}_{p} \tilde{p}\right)
$$

The implication is that the effective number of input parameters is reduced from $n$ to $d_{n}$.

\subsection{Efficient Basis Construction: Randomized Range Finder}

As illustrated in the previous section, the model is reducible if one can identify the subspace of essential features. Those features can be expressed as basis in mathematical language. Many methods have been proposed to compute the basis, e.g. Krylov subspace methods, balanced truncation methods, and proper orthogonal decomposition (see Ref. [1] for excellent review). Note that the basis construction requires the additional effort, e.g. solving minimization or eigenvalue problems with repetitive code executions. For the efficient basis construction, we adopted a range finder algorithm which is suitable with the parallel computing environment and does not requires any additional expensive calculations.

The range finder algorithm was originally devised for calculating a matrix low-rank approximation, of often very large matrices. The randomized algorithms utilize the random input perturbations to identify the sample range. For illustration of fundamental idea, consider finding a low rank approximation for a matrix $\mathbf{A}$ with an exact rank $k$. Pick $k$ randomly generated vectors $\vec{w}$ and form the matrix-vector product $\vec{z}=\mathbf{A} \vec{w}$ : 


$$
\vec{z}^{(i)}=\mathbf{A} \vec{w}^{(i)}, \quad i=1, \ldots, k
$$

Randomness guarantees that the vectors $\vec{w}^{(i)}$ for $i=1, \ldots, k$ as well as the vectors $\vec{z}^{(i)}$ for $i=1, \ldots, k$ are linearly independent. The vectors $\bar{z}^{(i)}$ for $i=1, \ldots, k$ are said to span the range of the matrix $\mathbf{A}$. To obtain an orthonormal basis for the range of the matrix $\mathbf{A}$, one can simply orthonormalize the samples, i.e. $\vec{z}^{(i)}$ for $i=1, \ldots, k$ [4].

Note that this algorithm does not require explicit access to the entries of the matrix $\mathbf{A}$; it only requires the action of $\mathbf{A}$ on a vector. The previous developments exploited this idea and extended to find the range of a general nonlinear model [3]. As illustrated above, the state solutions calculated with randomly perturbed input parameters would be independent, which implies that each sample has the information of the state variations. Therefore, the basis describing the state variations can be constructed by sampling and orthonormalizing the state samples. In the same sense, the first order derivatives at random input points would be independent and have the information of the effective parameter subspace. The basis for parameter-level reduction can be constructed by sampling and orthonormalizing the gradients. Thanks to randomness, the code executions for sampling the state solutions or the gradients can be parallelized with multi-core computers.

\subsection{Intersection Subspace for Data Transfer between Serially Coupled Codes}

We extend the previous approaches which were developed for a single physics model to problems with serially coupled models. Suppose two models as follows:

$$
\begin{array}{ll}
\text { Model A: } & R(\vec{p}, \vec{\Sigma})=\chi(\vec{p}) \\
\text { Model B: } & T(\vec{\Sigma}, \vec{\phi})=\zeta(\vec{\Sigma})
\end{array}
$$

where the state solutions of Model A are passed as inputs to Model $\mathbf{B}$. The primary goal is to identify the basis for the intermediate variables $\vec{\Sigma}$ preserving the overall coupled behaviors. Once the basis is calculated properly, the both models can be reduced-order transformed by using the ROM methods described in Section 2.2.

First, consider the intermediate variables in terms of Model A. The state-level reduction recasts the intermediate variables as:

$$
\vec{\Sigma} \rightarrow \mathbf{Q}_{\mathbf{A}} \mathbf{Q}_{\mathbf{A}}^{T} \vec{\Sigma}=\mathbf{Q}_{\mathbf{A}} \tilde{\Sigma}
$$

Note that the basis $\mathbf{Q}_{\mathrm{A}}$ is determined by the former model, i.e. Model $\mathbf{A}$. The implication of the reduced order modeling is to capture the dominant variations in state solutions. Then, the next model with transferred data can be re-written as follows: 


$$
T\left(\mathbf{Q}_{\mathbf{A}} \tilde{\Sigma}, \bar{\phi}\right)=\zeta\left(\mathbf{Q}_{\mathbf{A}} \tilde{\Sigma}\right)
$$

Next, consider the basis for reduced order modeling of Model $\mathbf{B}$. The parameter-level reduction transforms the transferred intermediate variables into:

$$
\mathbf{Q}_{\mathbf{A}} \tilde{\Sigma} \rightarrow \mathbf{Q}_{\mathbf{B}} \mathbf{Q}_{\mathbf{B}}^{T} \mathbf{Q}_{\mathrm{A}} \tilde{\Sigma}
$$

where $\mathbf{Q}_{\mathbf{B}}$ is the basis for sensitive components with respect to state solution or output responses. The reduced order modeling of Model $\mathbf{B}$ retains the parameter components with high sensitivity among the delivered data. This implies that the subspace of intermediate variables which would eventually contribute to the overall model behaviors is intersection of two subspaces, i.e. large variations and high sensitivities (Component 1 in Table 1).

Table 1. Characterization of Basis Component

\begin{tabular}{|l|c|c|c|}
\hline \multicolumn{2}{|c|}{} & \multicolumn{2}{c|}{ Model A } \\
\cline { 3 - 4 } & Large Variations & Small Variations \\
\hline \multirow{2}{*}{ Model B } & High Sensitivities & Component 1 & Component 2 \\
\cline { 2 - 4 } & Low Sensitivities & Component 3 & Component 4 \\
\hline
\end{tabular}

One can construct the basis for intersection subspace by performing two basis construction algorithms separately and combining them as in Eq. (12). In that case, the required code executions would be $d_{k}+\alpha d_{n}$ where $\alpha$ depends on the method of calculating derivatives, e.g. with the adjoint based perturbation theory, it would be 2 for criticality calculation and 3 for general responses. It is natural to think that the size of the intersection subspace would be smaller than any one of subspaces, i.e. $d_{\text {inter }} \leq \min \left(d_{k}, d_{n}\right)$. Therefore, if one can investigate the intersection subspace more directly, the required code executions would be reduced.

We propose the basis construction algorithm for the intersection subspace in two stages. In the first stage, the former model is executed with random input parameters to identify the patterns in state variations. We recommend to conduct this stage exhaustively as much as possible to avoid missing the components having small variations but very high sensitivity that eventually impact to the output responses (Component 2 in Table 1). The second stage is calculating the first order derivatives at random inputs by executing the next model. The basis for the intersection subspace can be filtered out by projecting the sensitivity samples onto the subspace of patterns and orthonormalizing them. With this approach, one can reduce the number of code executions for first order derivatives, i.e. proportional to $d_{\text {inter }}$ not to $d_{n}$. The procedure can be summarized as followings:

\footnotetext{
Proto-Algorithm: Intersection Subspace Basis Construction for Serially Coupled Codes

Given two coupled codes, this procedure computes the basis of the intersection subspace for intermediate variables transferred from the former code to the latter code.

Stage 1) Pattern Identification in Intermediate Variable Variations

- sample the intermediate variables by executing the former code with random inputs

- compute the basis for patterns by orthonormalizing samples
} 
Stage 2) Intersection Subspace Extraction

- $\quad$ sample the first order derivatives of pseudo responses ${ }^{1}$ with respect to intermediate variables at random points

- $\quad$ project the samples onto the subspace of patterns calculated in Stage 1

- compute the intersection subspace basis by orthornormalizing projected samples

\section{NUMERICAL TEST}

Assembly calculation can be considered as multi-physics problem. Resonance shielding calculations represent one physics code, necessary to calculate the effective multi-group crosssections, before passing them to the next physics code representing transport calculations. The transport code employs the effective cross-sections to solve for any responses of interest, e.g. keigenvalue, flux or few-group cross-sections. The depletion code takes the flux to calculate the nuclide density changes due to transmutation and depletion. This sequence of resonance transport - depletion calculations are repeated at each depletion step as shown in Figure 1.

We mainly focus on the part of resonance calculation and transport calculation, which correspond to Model A and Model B in Section 2.4, respectively. The effective multi-group cross-sections are considered as intermediate variables and the primary goal is to construct the basis for all possible variations during the entire depletion period under different operating conditions.

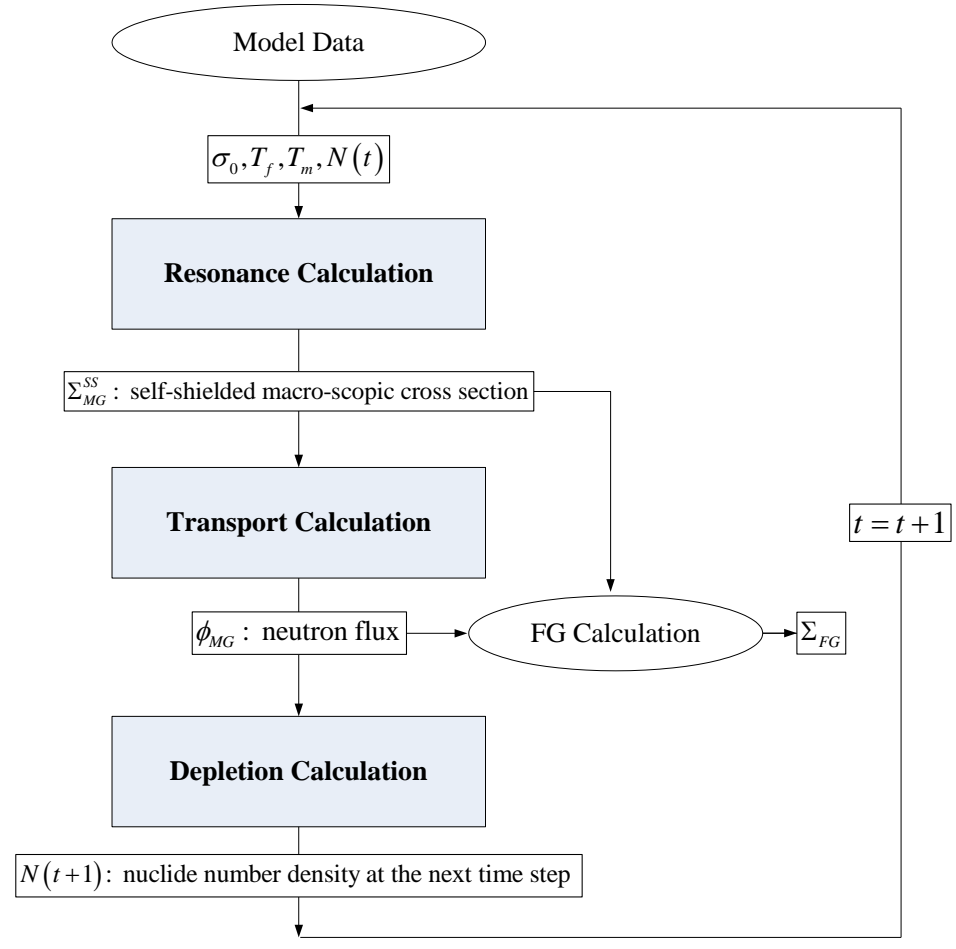

Figure 1. Schematics of Assembly Calculation

\footnotetext{
${ }^{1}$ A pseudo response is a random linear combination of the model's responses. See Ref [3] for more details on its construction.
} 
SCALE6.1 [5] is used for simulations and two different types of assembly models are considered (Figure 2): Peach Bottom Unit 2 Boiling Water Reactor (PB-2 BWR) [6] and Watts Bar Unit 2 Pressurized Water Reactor (WB-2 PWR) [7]. The applicability of our basis construction algorithm for highly nonlinear cases due to poison material burnout (Gd-155 \& Gd 157) and for cases with different fuel enrichments and control rod insertions are examined with PB-2 BWR and WB-2 PWR models, respectively. The original dimension of effective multi-group cross sections are 44,786 (914 nuclides with 49 energy group) and 184,240 (3,760 nuclides with 49 energy group), respectively ${ }^{2}$. Those models are depleted with burnup of $31.2 \mathrm{GWd} / \mathrm{MTIHM}$. To emulate the operating condition changes, temperatures and densities of fuel and moderator are varied by $\pm 10 \%$.

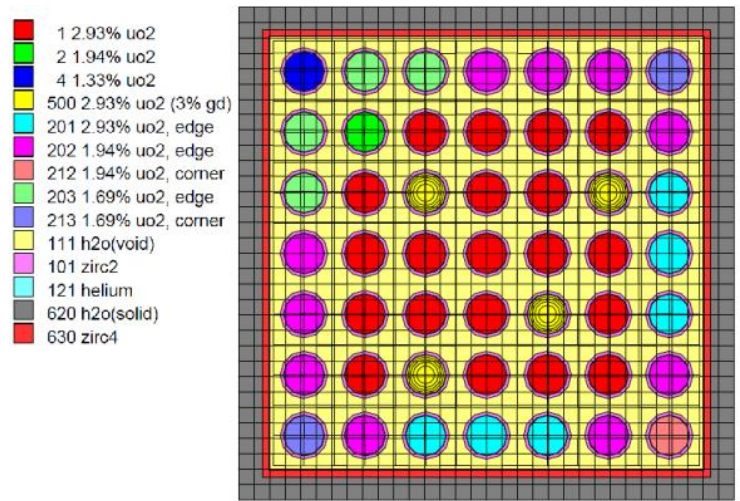

BWR Assembly Model (PB-2 BWR)

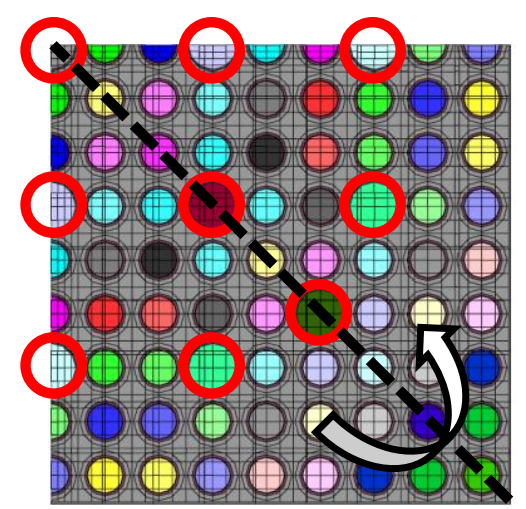

PWR Assembly Model (WB-2 PWR)

Figure 2. SCALE Assembly Model

Suppose that the models can be expressed as:

$$
\begin{aligned}
& \text { Model A (resonance caluclation): } \Delta \vec{\Sigma}=f(\Delta \vec{p}) \\
& \text { Model B (transport calculation): } \Delta k_{\text {eff }}=g(\Delta \vec{\Sigma})
\end{aligned}
$$

where

- $\Delta \vec{p}=\vec{p}-\vec{p}_{0}$ is the variations in system configurations, i.e. fuel/moderator temperatures or nuclide number densities,

- $\Delta \vec{\Sigma}=\vec{\Sigma}-\vec{\Sigma}_{0}$ is the effective macroscopic cross section changes,

- $\Delta k_{\text {eff }}=k_{\text {eff }}-k_{\text {eff }, 0}$ is a k-eigenvalue change,

- $\vec{p}_{0}, \vec{\Sigma}_{0}$ and $k_{\text {eff }, 0}$ are the reference values of system configurations, effective macroscopic cross sections and k-eigenvalue, respectively,

- $\quad f$ represents a resonance calculation (BONAMI)

- $\quad g$ represents a transport calculation (NEWT)

\footnotetext{
${ }^{2}$ the nuclides in different mixtures are counted as different nuclides though there are the same isotopes because they are depleted differently.
} 
The basis $\mathbf{Q}_{\mathrm{A}}$ of patterns in $\Delta \vec{\Sigma}$ can be calculated by (Stage 1):

1. sample $\Delta \vec{\Sigma}$ by running resonance calculations with randomly picked $\Delta \vec{p}$,

2. orthonormalizing samples by rank revealing decomposition (QR or SVD):

$$
\mathbf{Q}_{\mathbf{A}} \mathbf{R}=\left[\begin{array}{lll}
\Delta \vec{\Sigma}^{(1)} & \cdots & \Delta \bar{\Sigma}^{\left(d_{k}\right)}
\end{array}\right]
$$

Then, the basis for intersection subspace can be extracted by (Stage 2):

1. sample $\frac{\partial k_{\text {eff }}}{\partial \vec{\Sigma}}$ by solving transport models (forward and adjoint) with randomly picked $\vec{\Sigma}$,

2. project the sampled sensitivity profiles onto the subspace spanned by $\mathbf{Q}_{\mathrm{A}}$ and orthonormalizing samples by rank revealing decomposition (QR or SVD): $\mathbf{Q R}=\mathbf{Q}_{\mathbf{A}} \mathbf{Q}_{\mathbf{A}}^{T}\left[\left.\left.\frac{\partial k_{\text {eff }}}{\partial \vec{\Sigma}}\right|^{(1)} \cdots \frac{\partial k_{e f f}}{\partial \vec{\Sigma}}\right|^{\left(d_{\text {inter }}\right)}\right]$.

Note that in step 1 of Stage 2, $\vec{\Sigma}$ is picked independently from Stage 1, i.e. directly random sampled. To ensure that the constructed basis represents all essential features of assembly calculations appropriately and to estimate the error due to reduced order transformation, the basis is verified with three approaches: singular value spectrum, norm of discarded component and keigenvalue changes due to discarded component.

First of all, the singular value spectrums are compared. Note that the randomized range finder algorithm extracts the information from each sample. Therefore, the singular values can be interpreted as the importance of each sample:

$$
\mathbf{U D V}^{T}=\left\{\begin{array}{lll}
{\left[\begin{array}{lll}
\Delta \vec{\Sigma}^{(1)} & \cdots & \Delta \vec{\Sigma}^{(i)}
\end{array}\right]} & \text { for single subspace (pattern) } \\
\mathbf{Q}_{\mathbf{A}} \mathbf{Q}_{\mathbf{A}}^{T}\left[\frac{\left.\frac{\partial k_{e f f}}{\partial \vec{\Sigma}}\right|^{(1)}}{} \cdots\right. & \left.\left.\frac{\partial k_{e f f}}{\partial \vec{\Sigma}}\right|^{(i)}\right] & \text { for intersection subspace }
\end{array}\right.
$$

where $\mathbf{U}$ and $\mathbf{V}$ are left and right singular vectors, respectively and $\mathbf{D}$ is the diagonal matrix whose entries are singular values [8]. As can be seen in Figure 3, the singular value spectrum of the intersection subspace is decaying faster than the one of the single subspace. This shows that the intersection subspace can confine the effective multi-group cross-sections into smaller dimension at a given precision (fixed-precision problem) or with a given subspace size, the intersection subspace approach provides more optimal basis (fixed-size problem). 


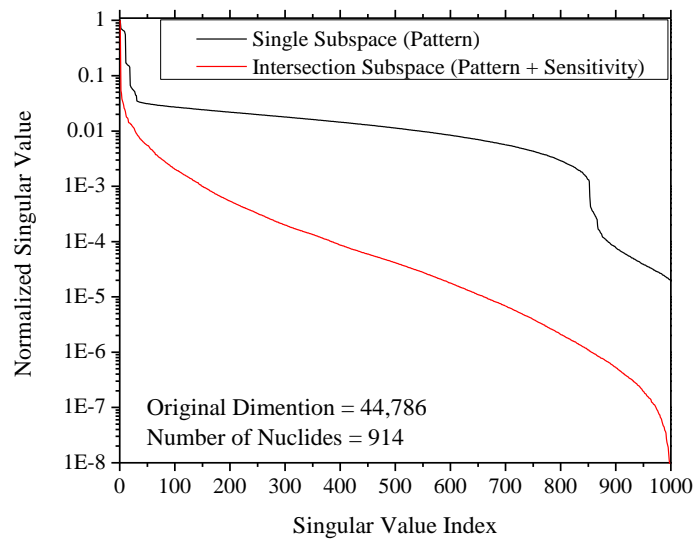

BWR Assembly Model (PB-2 BWR)

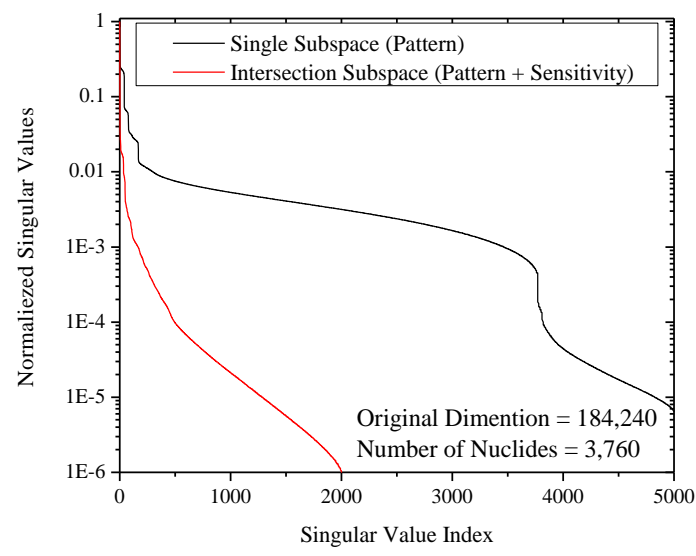

PWR Assembly Model (WB-2 PWR)

\section{Figure 3. Singular Value Spectrum of Random Samples}

Viewing the macroscopic cross sections as outputs of resonance calculations, the approximation error can be directly estimated by calculating the discarded components in the macroscopic cross section variations due to resonance calculations with perturbed number densities or temperatures/densities:

$$
\Delta \vec{\Sigma}^{\perp}=\left\{\begin{array}{cl}
\Delta \vec{\Sigma}-\mathbf{Q}_{\mathbf{A}} \mathbf{Q}_{\mathbf{A}}^{T} \Delta \vec{\Sigma}=\left(\mathbf{I}-\mathbf{Q}_{\mathbf{A}} \mathbf{Q}_{\mathbf{A}}^{T}\right) \Delta \vec{\Sigma} & \text { for single subspace (pattern) } \\
\Delta \vec{\Sigma}-\mathbf{Q Q}^{T} \Delta \vec{\Sigma}=\left(\mathbf{I}-\mathbf{Q} \mathbf{Q}^{T}\right) \Delta \vec{\Sigma} & \text { for intersection subspace }
\end{array}\right.
$$

In Figure 4, the root mean square of discarded component (i.e. not captured by constructed basis) of effective multi-group cross-sections are compared. For basis of patterns, the discarded components decrease as the basis size increases, which means that most of variations are captured. On the other hand, one can see that much more components are filtered out with intersection approach by considering sensitivity.

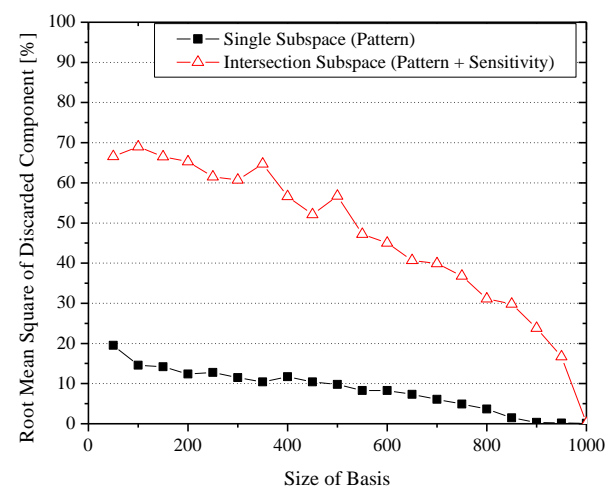

BWR Assembly Model (PB-2 BWR)

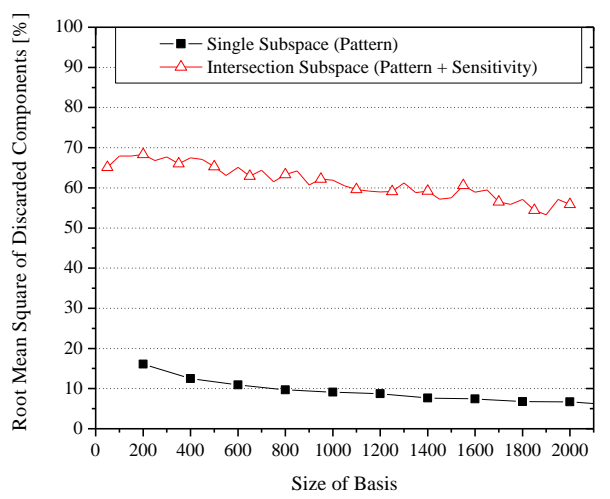

PWR Assembly Model (WB-2 PWR)

Figure 4. Root Mean Square of Discarded Components 
Considering the macroscopic cross sections as inputs of transport calculations, k-eigenvalue changes due to discarded components can be calculated to estimate the error due to approximation:

$$
0 \approx \Delta k_{\text {eff }}=g\left(\Delta \vec{\Sigma}^{\perp}\right)=\left\{\begin{array}{cl}
g\left(\left(\mathbf{I}-\mathbf{Q}_{\mathbf{A}} \mathbf{Q}_{\mathbf{A}}^{T}\right) \Delta \vec{\Sigma}\right) & \text { for single subspace (pattern) } \\
g\left(\left(\mathbf{I}-\mathbf{Q} \mathbf{Q}^{T}\right) \Delta \vec{\Sigma}\right) & \text { for intersection subspace }
\end{array}\right.
$$

If the basis is properly constructed, the k-eigenvalue changes should be very small. This approach may be most rigorous way to verify the basis. To test this, macroscopic cross section variations for an assembly model at every depletion calculation steps are collected and projected to calculate the discarded component. The transport calculation (NEWT) is performed with those discarded components to check the k-eigenvalue change. In Figure 5, the k-eigenvalue changes due to discarded components are compared. With intersection approach, the subspace size of 800 and 2000 would be sufficient for PB-2 BWR and WB-2 PWR, respectively, while those are not enough for single subspace approach. Though not included in this manuscript, the basis is verified also with respect to other responses, e.g. scalar fluxes and assembly-homogenized fewgroup cross-sections. Considering the precision of ENDF cross-section library and transport solver convergence criteria ( $10^{-5}$ for PB-2 BWR and $10^{-4}$ for WB-2 PWR), the required size of basis could be determined and summarized in Table 2.

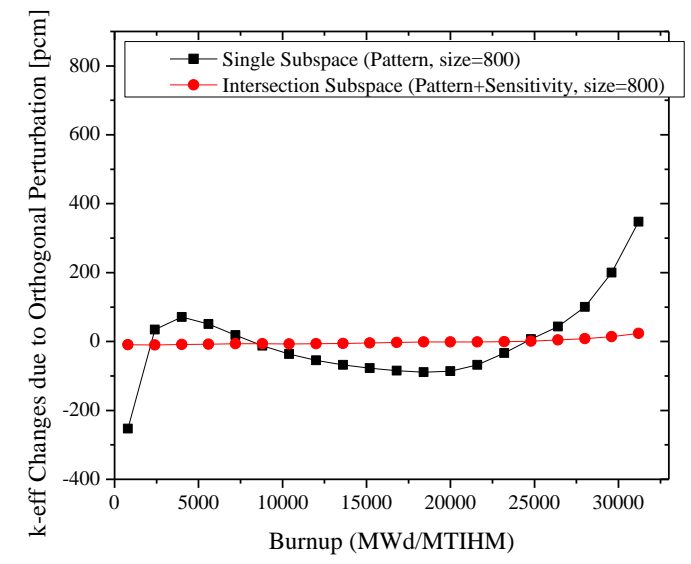

BWR Assembly Model (PB-2 BWR)

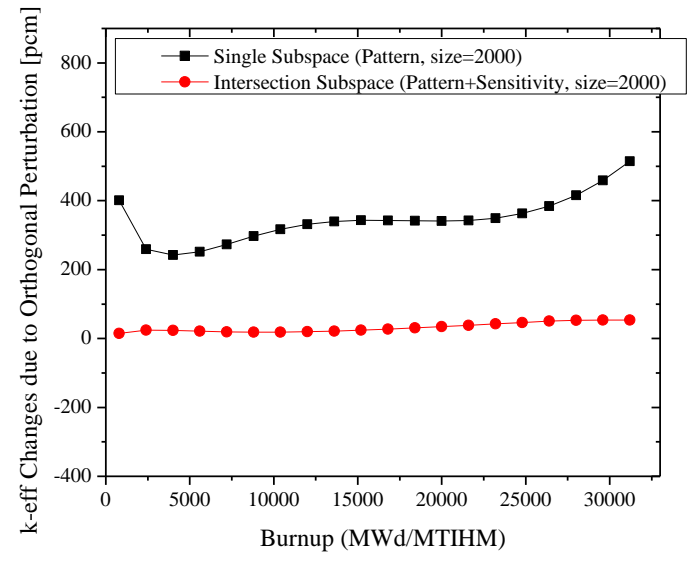

PWR Assembly Model (WB-2 PWR)

Figure 5. k-eigenvalue changes due to Discarded Components (Basis Size=800 for PB-2 BWR and 2000 for WB-2 PWR)

Table 2. Summary of Test Result

\begin{tabular}{|c|c|c|}
\hline & PB-2 BWR & WB-2 PWR \\
\hline Original Dimension & 44,786 & 184,240 \\
\hline $\begin{array}{c}\text { Reduced Dimension } \\
\text { (single subspace, pattern })\end{array}$ & $\begin{array}{c}1,000 \\
(2.23 \% \text { of original dim. })\end{array}$ & $\begin{array}{c}5,000 \\
(2.71 \% \text { of original dim. })\end{array}$ \\
\hline $\begin{array}{c}\text { Reduced Dimension } \\
\text { (intersection sub., pattern+sensitivity })\end{array}$ & $\begin{array}{c}800 \\
(1.79 \% \text { of original dim. })\end{array}$ & $\begin{array}{c}2,000 \\
(1.09 \% \text { of original dim. })\end{array}$ \\
\hline
\end{tabular}




\section{CONCLUSION}

We developed the efficient reduced order modeling algorithm for serially coupled code system and demonstrated it on assembly calculations for fuel loading pattern optimization, i.e. assemblywide resonance/transport/depletion calculations under different temperature and density conditions and material compositions. While each physics code is independently reducible, by combining the basis from each code using the idea of subspace intersection, further reduction can be achieved. Normally, as more codes are coupled, the simulation would become more complicated and computationally intensive, which makes many engineering analysis impractical. In such situations, the intersection approach would be very useful to alleviate the computational burdens because more reduction is expected as more codes are considered.

\section{ACKNOWLEDGMENTS}

This work is supported by the Consortium for Advanced Simulation of Light Water Reactors (www.casl.gov), an Energy Innovation Hub (http://www.energy.gov/hubs) for Modeling and Simulation of Nuclear Reactors under U.S. Department of Energy Contract No. DE-AC05$00 \mathrm{OR} 22725$.

\section{REFERENCES}

1. A.C. Antoulas, Approximation of Large-Scale Dynamical Systems, Book Series: Advances in Design and Control, DC 06, SIAM, Philadelphia, (2005).

2. Y.S. Bang, C.J. Wang, and H.S. Abdel-Khalik, "State-Based Adjoint Method for Reduced Order Modeling”, Transport Theory and Statistical Physics, Volume 41, Issue 1-2, pp.101132, (2012).

3. Y.S. Bang, H.S. Abdel-Khalik, and J.M. Hite, "Hybrid Reduced Order Modeling Applied to Nonlinear Models”, International Journal for Numerical Methods in Engineering, Volume 91, Issue 9, pp.929-949, (2012).

4. N. Halko, P. Martinsson, and J. Tropp, "Finding structure with randomness: Probabilistic algorithms for constructing approximate matrix decompositions", SIAM Review, Volume 53, Number 2, pp.217-288, (2011).

5. SCALE 6.1: A Modular Code System for Performing Standardized Computer Analyses for Licensing Evaluations, ORNL/TM-2005/39, Version 6.1, Oak Ridge National Laboratory, Oak Ridge, Tennessee, (2011).

6. K.N. Ivanov, T.M. Beam, and A.J. Baratta, Pressurised Water Reactor Main Steam Line Break (MSLB) Benchmark, Volume I: Final Specifications, US Nuclear Regulatory Commission/OECD Nuclear Energy Agency, NEA/NSC/DOC(99)8, (1999).

7. J.C. Wagner, and C.V. Parks, Parametric Study of the Effect of Burnable Poison Rods for PWR Burnup Credit, NUREG/CR-6761 (ORNL-TM-2000/373), ORNL, March, (2002).

8. G.H. Golub and C.F. Van Loan, Matrix Computations (3rd ed.), John Hopkins, Baltimore, (1996). 